\title{
Exploring the complexity of the childhood trait-psychopathology association: Continuity, pathoplasty, and complication effects
}

\author{
MARLEEN DE BOLLE, BARBARA DE CLERCQ, ELIEN DE CALUWÉ, AND LIZE VERBEKE \\ Ghent University
}

\begin{abstract}
Four different models have been generally proposed as plausible etiological explanations for the relation between personality and psychopathology, namely, the vulnerability, complication, pathoplasty, and spectrum or continuity model. The current study entails a joint investigation of the continuity, pathoplasty, and complication models to explain the nature of the associations between early maladaptive traits and psychopathology over time in 717 referred and community children (54.4\% girls), aged from 8 to 14 years. Across a 2-year time span, maladaptive traits and psychopathology were measured at three different time points, thereby relying on comprehensive and age-specific dimensional operationalizations of both personality symptoms and psychopathology. The results demonstrate overall compelling evidence for the continuity model, finding more focused support for pathoplasty and complication effects for particular combinations of personality symptoms and psychopathology dimensions. As expected, the continuity associations were found to be more robust for those personality-psychopathology associations that are conceptually closer, such as the emotional instability/introversion-internalizing problems association and the disagreeableness-externalizing problems association. Continuity associations were also stronger when personality was considered from a maladaptive rather than from a general trait perspective. The implication of the findings for the treatment of psychopathology and personality symptoms are briefly discussed.
\end{abstract}

Although clinical disorders and personality pathology have been traditionally conceived as distinct groups of pathology (Clark, 2005), empirical evidence does generally not support this conceptual bifurcation. More specifically, there has been a historical belief that personality disorders, in contrast to clinical disorders, reflect enduring patterns that are relatively stable over time. Recent longitudinal studies, however, have demonstrated a natural plasticity of personality symptoms both in adulthood (Cohen, Crawford, Johnson, \& Kasen, 2005; Lenzenweger, 1999; Livesley, 2005; Skodol et al., 2005; Zanarini, Frankenburg, Hennen, Reich, \& Silk, 2005) and at younger ages (De Clercq, Van Leeuwen, Van den Noortgate, De Bolle, \& De Fruyt, 2009). Furthermore, it has been demonstrated that clinical symptoms and Axis I disorders display a higher stability over time than initially assumed (Briggs-Gowan et al., 2003; Costello, Mustillo, Erkanli, Keeler, \& Angold, 2003; Roza, Hofstra, van der Ende, \& Verhulst, 2003). In addition, a mass of literature reports on the substantial overlap between personality and psychopathology in adults (Widiger, 2003) and children (Tackett, 2006), including shared genetic etiological factors (Bienvenu, Hettema, Neale, Prescott, \& Kendler, 2007; Krue-

Partial support of the preparation of this manuscript was provided by Postdoctoral Fellowship 1200613N (to M.D.B.) from the National Research Fund Flanders.

Address correspondence and reprint requests to: Marleen De Bolle, Department of Developmental, Personality and Social Psychology, Faculty of Psychology and Educational Sciences, Ghent University, Henri Dunantlaan 2, Ghent 9000, Belgium; E-mail: Marleen.DeBolle@UGent.be. ger, Markon, Patrick, \& Iacono, 2005). These research findings all suggest that personality and psychopathology are closely interconnected, given their similar nature and developmental course over time. Much is to be studied, however, to unravel their complex longitudinal interrelations from childhood onward. Delineating these trait-psychopathology connections from the various etiological models makes it possible to map causally oriented pathways of maladjustment toward adulthood (De Clercq et al., 2009). The current study aims to explore the etiology of these associations between early maladaptive traits and psychopathology over time, relying on comprehensive and age-specific dimensional conceptualizations of maladaptive personality and psychopathology.

\section{Etiological Models of Trait-Psychopathology Associations}

Four different models have been generally proposed as plausible etiological explanations for the relations between personality and psychopathology. According to the predisposition/vulnerability model, the presence of particular personality traits increases the probability of developing a clinical disorder, whereas the complication/scar model posits that an existing clinical disorder may cause changes in personality. The pathoplasty/exacerbation model postulates that cooccurring general personality traits and clinical disorders or symptoms may have an independent etiology and onset, but personality can influence the course or manifestation of these clinical disorders or symptoms. Finally, the spectrum model 
assumes that personality traits and clinical disorders form a spectrum ranging from general traits to subclinical characteristics to full-blown psychopathology because of shared underlying etiological factors (Krueger \& Tackett, 2003; Mineka, Watson, \& Clark, 1998). The latter model is closely connected to the continuity model, referring to the systematic phenomenological covariation of personality and psychopathology within and across time, without pronouncing upon etiology or causality of this covariation. From this perspective, the continuity hypothesis can be considered a prerequisite condition of the spectrum hypothesis (De Bolle, Beyers, De Clercq, \& De Fruyt, 2012). More recently, integrative models have been proposed that combine these etiological models into an overarching framework on the links among general personality, personality pathology, and psychopathology (i.e., Clark, 2005; Krueger \& Tackett, 2003). These integrative models assume that the empirical connection between personality and psychopathology is mostly consistent with the continuity/spectrum hypothesis, and that complication, pathoplasty, and vulnerability effects further extend and augment this continuity/spectrum model.

De Bolle et al. (2012) and Tackett (2006) provided a detailed and comprehensive overview of the evidence in support of the proposed etiological models that exist on the traitpsychopathology association in preadulthood. They concluded that various studies empirically underscored each of these models, supporting the idea that these etiological models are not mutually exclusive, and that different models perhaps explain different types of psychopathology (Dolan-Sewell, Krueger, \& Shea, 2001). The literature reviewed by Tackett (2006) has, however, mainly been provided by relying on only one single model, without controlling for the effects of other etiological associations. This methodological choice may have resulted in biased findings and complicates the transparency of the conclusions culled from these designs. From a more rigorous perspective, De Bolle et al. (2012) showed that the effects of the continuity model preponderate the general trait-psychopathology relation in childhood and reported more focused evidence for the pathoplasty and complication models, depending on the particular personality-psychopathology association under consideration. More specifically, they found continuity associations between internalizing and externalizing psychopathology, on the one hand, and each of the personality dimensions, on the other hand. In addition to these continuity associations, they reported complication effects from internalizing problems on emotional instability and conscientiousness, and from externalizing problems on extraversion, benevolence, and conscientiousness. Pathoplasty effects were found for benevolence on both internalizing and externalizing problem behavior and for extraversion on externalizing problem behavior. These findings highlight the dimensional nature of traits and psychopathology, suggesting that they primarily need to be understood as continuous and related constructs, although they both also represent a unique set of characteristics that appear to have a reciprocal influence from a more causally oriented pathway.
However, the literature discussed above addressed the associations between child and adolescent psychopathology and personality from a general trait variation perspective. A closely related and essential research question pertains to the relation between childhood maladaptive traits and psychopathology across time. Given the vast amount of evidence supporting the similar dimensional nature of personality and psychopathology (Krueger, 2005), it may be interesting to see whether similar etiological relations with constructs of psychopathology exist across the spectrum of general and pathological trait variance. De Clercq et al. (2009) illustrated that childhood maladaptive traits and broad dimensions of internalizing and externalizing psychopathology generally show similar longitudinal patterns in terms of shape and change over time in a community-based childhood sample, providing indirect support for the continuity model. However, this study did not address the link between (change patterns of) maladaptive traits and psychopathology. To the best of our knowledge, only De Clercq, Van Leeuwen, De Fruyt, Van Hiel, and Mervielde (2008) examined the associations between childhood maladaptive traits and psychopathology. They found particular strong correlations between internalizing problems and emotional instability and introversion and between externalizing problems and disagreeableness, even after controlling for item-overlap between the constructs of maladaptive personality and psychopathology. Given the cross-sectional design of this study, however, no conclusions could be drawn on the nature (e.g., continuity association, pathoplasty associations, or complication associations) of these personality-psychopathology associations. An empirical test of these hypotheses may help to unravel the specific mechanisms that underlie the development of early maladaptation, and may further define how these processes parallel the adaptive trait-psychopathology development. The current study will, therefore, simultaneously investigate the continuity, pathoplasty, and complication models, ${ }^{1}$ using three-wave data on personality pathology and psychopathology from community and referred children and adolescents. This approach enables disentanglement of the different models and pronouncement upon the specific applicability of each of these models to explain the nature of the maladaptive traits-psychopathology associations at a young age.

\section{Conceptualization of Personality Dysfunction and Psychopathology}

There has long been compelling evidence that childhood clinical disorders can be hierarchically organized in the

1. The predisposition model was not evaluated because personality was not measured systematically before the onset of psychopathology symptoms. A proxy investigation of the predisposition model would be to investigate personality differences between individuals from the population sample who developed clinical syndrome scores at T2 or T3 and individuals with nonclinical scores at all three measurement occasions. However, because of the very low base rates of individuals in the community sample scoring beyond clinical cutoffs for internalizing or externalizing problems, such analyses were not possible with the current sample. 
two-dimensional frame of internalizing and externalizing disorders (Achenbach, 1991). More recently, these two broad super factors of internalization and externalization were also found to be relevant for integrating adult psychopathology (Krueger, 1999). Together, the literature suggests that clinical and more trait-related pathology can be meaningfully captured by means of dimensional models that generalize across the life span.

Furthermore, there is a general consensus on the basic dimensional structure of maladaptive personality in adulthood, with emotional dysregulation versus emotional stability, extraversion versus introversion, antagonism versus compliance, and constraint versus impulsivity as the four broad maladaptive trait dimensions (Widiger \& Simonsen, 2005). De Clercq, De Fruyt, Van Leeuwen, and Mervielde (2006) reported that the basic dimensional structure of early maladaptive trait characteristics can be conceptualized along four broad trait factors, labeled as emotional instability, introversion, compulsivity, and disagreeableness. Substantial empirical evidence has indicated that this childhood dimensional model of personality pathology shows a striking similarity to its adult counterpart with respect to structure, content, and development (De Clercq et al., 2009). In addition, it has been demonstrated that these four maladaptive trait dimensions are extreme variants of four of the five five-factor model (McCrae \& Costa, 2008) personality dimensions, together tapping four trait continua: emotional stability/emotional instability, extraversion/introversion, conscientiousness/ compulsivity, and agreeableness/disagreeableness (Widiger \& Simonsen, 2005). Integrating maladaptive and general personality trait dimensions within a five-factor model framework provides a basis for meaningfully comparing and relating personality-psychopathology associations from a general versus pathological trait perspective.

\section{Present Study}

The present study aims to simultaneously investigate the continuity, pathoplasty, and complication models as plausible explanations of the nature of maladaptive personality-psychopathology associations at a young age. In line with the theoretical considerations of Clark (2005) and Krueger and Tackett (2003) and the empirical findings of De Bolle et al. (2012), we hypothesize to find substantial support for the continuity model and more focused support for the complication and pathoplasty models. More specifically, consistent with other studies in the field (Lilienfeld, 2003), we expect that emotional instability will be related to both internalizing and externalizing behaviors, because this personality dimension is mainly responsible for the frequently found correlation between the internalizing and externalizing dimensions. Therefore, we expect to find significant associations between emotional instability, on the one hand, and internalizing and externalizing psychopathology, on the other hand, both within and across time. Furthermore, in line with the findings of De Bolle et al. (2012), we expect to find that the maladaptive counterparts of agreeableness (i.e., disagreeableness) will be more strongly related to externalizing behavior, whereas the maladaptive variants of emotional stability (i.e., emotional instability) and extraversion (i.e., introversion) will be more strongly related to internalizing behavior. Based on the findings of De Clercq et al. (2006), we expect that compulsivity would be more strongly associated with internalizing than with externalizing psychopathology. Finally, we expect to find more focused support for the complication and pathoplasty models, depending on the specific personality-psychopathology relation under consideration.

Maladaptive traits and psychopathological constructs are even more closely related from a conceptual point of view than the general trait-psychopathology constructs because they both describe aspects of maladaptation at the extreme end of behavioral, emotional, and cognitive dimensions. Therefore, we hypothesize that maladaptive trait-psychopathology associations differ from the general trait-psychopathology associations in terms of strength, but not in terms of direction.

\section{Method}

\section{Participants and procedure}

In the current study, the same samples as in De Bolle et al. (2012) were used. ${ }^{2}$ More specifically, data of both a referred and a community sample of children were collected in the course of the Personality and Affect Longitudinal Study. Participants of both samples were recruited at baseline at Time 1 (T1) by third-year undergraduate psychology students of Ghent University and follow-up assessments were organized via mail at 1 year at Time 2 (T2) and 2 years at Time 3 (T3) after initial assessment. Data collection was approved by the Ghent University Ethical Review Board. All participants were assured that the information would be treated as confidential and would only serve research purposes. Written informed consent was obtained from all mothers and children. The combined sample consists of 717 children (54.4\% girls), aged from 8 to 14 years $(M=10.74, S D=1.37)$.

Community sample. Students were instructed to recruit a child from the general population in their own family or social environment, after detailed information on study aims, procedure, and ethics of data collection was provided. Given that Ghent

2. The study of De Bolle et al. (2012) investigated empirical evidence for the pathoplasty, complication, and continuity models to explain the nature of personality-psychopathology relations from a general trait perspective. That is, this study focused on the associations between general personality dimensions and psychopathology across time. By contrast, the current study examines the nature of the personality-psychopathology relations from a maladaptive trait perspective, thereby also considering the pathoplasty, complication, and continuity models as possible explanatory models. As such, these studies partly share the same data (i.e., data on psychopathology, as measured by the CBCL), but include different data on personality (i.e., data on general versus maladaptive personality, as measured by the HiPIC and DiPSI, respectively). To maximize the comparability of the findings, the current study adopts a very similar data analytic approach as in De Bolle et al. (2012). 
University students originate from different regions in Flanders, this sampling method guarantees an adequate geographical distribution of the current sample ( $n=571$; $55 \%$ girls; mean age $=10.70, S D=1.23$ ). All children were between 8 and 14 years old, with Dutch as the native language of both mother and child. Exclusion criteria included having a mental handicap and/or having any physical constraints or disabilities. Students visited the families at home and asked mother and child to complete a set of questionnaires in two separate rooms, to ensure that their independent opinion was assessed. Students were instructed not to assist participants and were only allowed to explain the meaning of item wordings that were not clear to the child or the mother. The response ratio was $71.8 \%$ for the $\mathrm{T} 2$ and $65.5 \%$ for the $\mathrm{T} 3$ data.

Referred sample. Referred children ( $n=146 ; 52.1 \%$ girls; mean age $=10.87, S D=1.84$ ) were recruited via a broad range of general mental health services, listed on an online directory with registered providers of psychological care in Flanders. The first author coordinated the selection of mental health services in order to ensure a broad geographical coverage across Flanders, and provided all contact information of a particular service to each of the students. Students were instructed to contact one of the treating psychologists of childhood internalizing problems by telephone and to explain the study aims, the procedures, and the ethics of data collection. When psychologists agreed to participate, the first child (and his parents) on the psychologist's appointment schedule that qualified for the study was asked to take part in the current research. Parents and child were given a package of questionnaires, consent forms, and information letters, and were asked to return the completed questionnaires at the next appointment in a sealed envelope, which was directly forwarded to the researchers. The response ratio was $82.9 \%$ and $68.5 \%$ for $\mathrm{T} 2$ and $\mathrm{T} 3$, respectively.

Parallel to the population sample, Dutch was the native language of both mother and child, and a condition of a mental or physical handicap served as an exclusion criterion. The primary reason for referral was the presence of anxiety symptoms (20.7\%), depressive symptoms (14.5\%), grief or emotional problems due to parental divorce $(11.3 \%)$, behavioral problems $(10.7 \%)$, personality or identity problems (7.6\%), developmental disorders $(6.9 \%)$, psychosomatic symptoms $(5.0 \%)$, learning problems $(5.0 \%)$, social problems $(3.8 \%)$, attention or concentration problems $(2.5 \%)$, sleeping problems $(1.9 \%)$, eating problems $(1.3 \%)$, enuresis problems $(1.3 \%)$, or self-mutilation/suicide attempts $(0.6 \%)$. For $6.9 \%$ of the children, the initial reason for counseling was not available. At T1, 11.3\% of the referred children were situated in the intake phase; $20.3 \%$ received psychological advice or further orientation, $63.2 \%$ received psychotherapy, and $5.3 \%$ visited the mental health center because of persisting or recurring problems.

\section{Measures}

Psychopathology. Mothers completed the Dutch version of the Child Behavior Checklist (CBCL; Verhulst, Van der
Ende, \& Koot, 1996) at each assessment period to evaluate their child's behavioral and emotional problems in the past 6 months. The CBCL contains 113 items that are scored on a 3 -point scale $(0=$ not true, $1=$ somewhat or sometimes true, and $2=$ very or often true). Two empirically derived broadband scales can be computed: internalizing problems (including the anxiety/depression, somatic complaints, and social withdrawal syndrome scales) and externalizing problems (including the delinquent behavior and aggressive behavior syndrome scales). The Cronbach $\alpha$ values for internalizing and externalizing problems were 0.91 and 0.90 at $\mathrm{T} 1$, 0.91 and 0.92 at $\mathrm{T} 2$, and 0.92 and 0.94 at T3.

Personality symptoms. Mothers were asked to complete the Dimensional Personality Symptom Item Pool (DIPSI; De Clercq, De Fruyt, \& Mervielde, 2003) at T1, T2, and T3 to assess their child's personality symptoms. The DIPSI consists of 172 concrete maladaptive trait items, empirically structured in 27 reliable and homogeneous lower level facets that are hierarchically organized in a replicable four-dimensional higher order structure: disagreeableness (including extreme low-end variants of benevolence such as dominance/ egocentrism and irritable/aggressive traits), emotional instability (referring to both anxious and depressive traits, and also including a dependency component), introversion (describing extreme low-end variants of extraversion, such as withdrawn traits and shyness), and compulsivity (including the high extremes of conscientiousness traits such as perfectionism and extreme order). All DIPSI items are scored on a 5-point Likert scale $(1=$ hardly characteristic, $5=$ very characteristic). Good internal consistencies were obtained for the DIPSI domains: $\alpha$ s ranged from 0.80 (compulsivity) to 0.93 (emotional instability and introversion) at $\mathrm{T} 1$, from 0.84 (compulsivity) to 0.94 (emotional instability) at $\mathrm{T} 2$, and from 0.86 (compulsivity) to 0.95 (emotional instability and introversion) at $\mathrm{T} 3$.

\section{Statistical analyses}

Evidence for the pathoplasty, complication, and continuity models will be investigated simultaneously (see Figure 1 for a conceptual multivariate latent change model [LCM] linking internalizing and externalizing problems to emotional instability across time). Because the within-time correlation between personality and psychopathology at initial assessment may also reflect persisting vulnerability effects, we will only consider correlated change between personality and psychopathology as empirical evidence for the continuity model.

Preparatory to fitting the LCMs, confirmatory factor analysis (CFA) was conducted to fit the measurement models and, as such, estimate latent scores for the constructs of interest. To obtain the latent or factor scores of the maladaptive personality factors, a four-factor model with the DIPSI marker facets as indicators (De Clercq et al., 2006) was estimated for each measurement occasion separately. Likewise, to estimate the factor scores of internalizing and externalizing problems, 


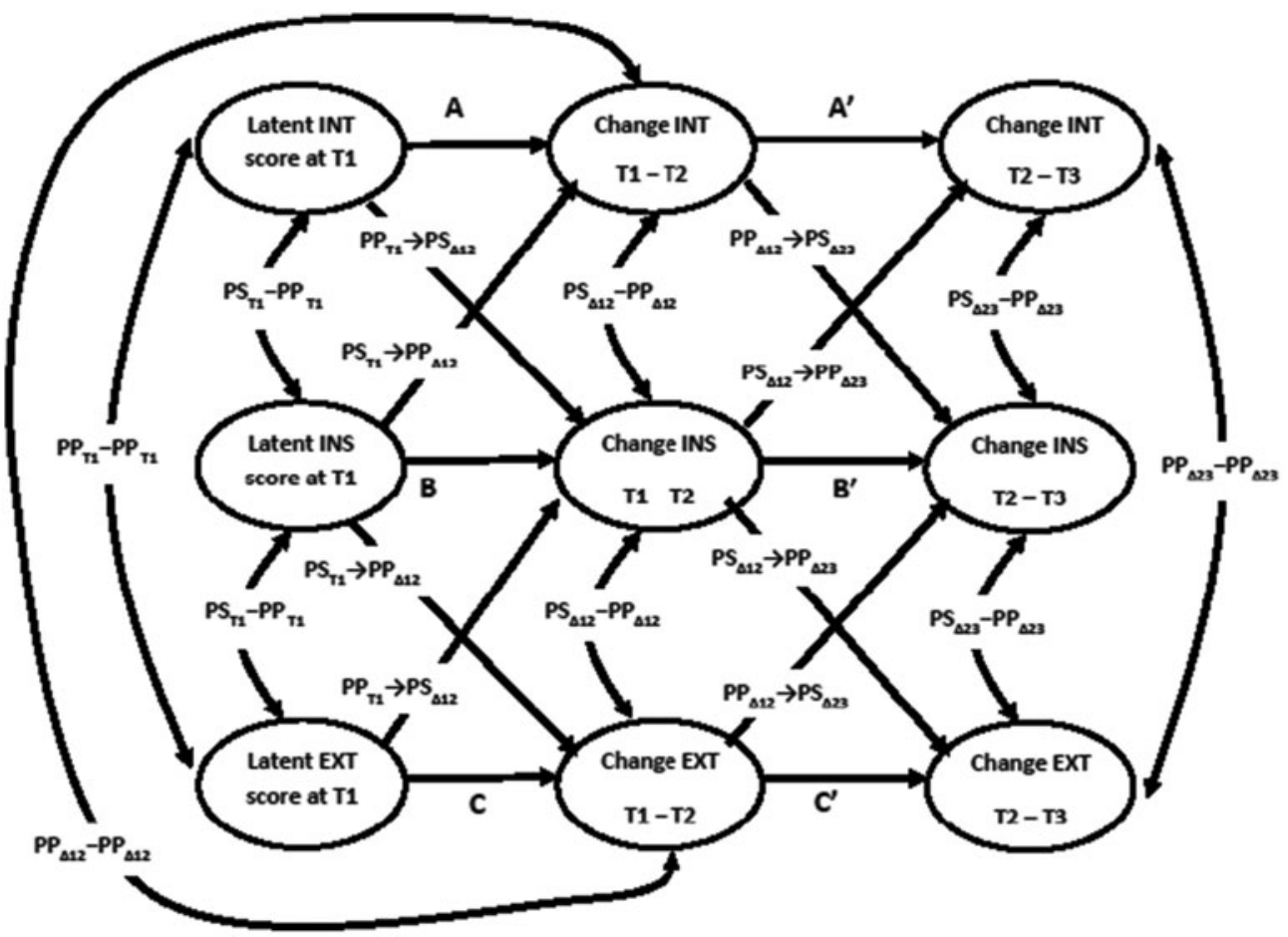

Figure 1. Conceptual latent change model linking internalizing and externalizing problems to emotional instability. T1, Time 1 first measurement occasion; T2, Time 2 second measurement occasion; T3, Time 3 third measurement occasion; INT, internalizing problems; EXT, externalizing problems; INS, emotional instability; PS, personality symptoms (i.e., INS in the present example); PP, psychopathology (i.e., INT and EXT in the present example). Paths A, A', B, B', C, and $\mathrm{C}^{\prime}$ represent the autoregressive paths for changes in INT, INS, and EXT, respectively. For example, A reflects whether children's INT level at T1 predicts change in INT from T1 to T2, whereas $\mathrm{A}^{\prime}$ reflects whether change in INT from T1 to T2 is predictive of change in INT from T2 to T3. Paths $\mathrm{PS}_{\mathrm{T} 1}-\mathrm{PP}_{\mathrm{T} 1}, \mathrm{PS}_{\triangle 12}-\mathrm{PP}_{\triangle 12}$, and $\mathrm{PS}_{\triangle 23}-\mathrm{PP}_{\triangle 23}$ reflect the continuity hypothesis, because they encompass that (changes in) INT and/or EXT and INS are related across time. These paths are corrected for covariation between INT and EXT (i.e., paths $\mathrm{PP}_{\mathrm{T} 1}-\mathrm{PP}_{\mathrm{T}}, \mathrm{PP}_{\triangle 12}-\mathrm{PP}_{\triangle 12}$, and $\mathrm{PP}_{\triangle 23}-\mathrm{PP}_{\triangle 23}$ ). However, because the $\mathrm{PS}_{\mathrm{T} 1}-\mathrm{PP}_{\mathrm{T} 1}$ path may reflect the persistence of a vulnerability effect, only $\mathrm{PS}_{\triangle 12}-\mathrm{PP}_{\triangle 12}$ and $\mathrm{PS}_{\triangle 23}-\mathrm{PP}_{\triangle 23}$ will be considered as evidence of the continuity model. Paths $\mathrm{PP}_{\mathrm{T} 1} \rightarrow \mathrm{PS}_{\triangle 12}$, and $\mathrm{PP}_{\triangle 12} \rightarrow \mathrm{PS}_{\triangle 23}$ are informative about the complication hypothesis, reflecting an effect of INT/EXT or a change in INT/EXT on subsequent change in INS. Paths $\mathrm{PS}_{\mathrm{T} 1} \rightarrow \mathrm{PP}_{\triangle 12}$, and $\mathrm{PS}_{\triangle 12} \rightarrow \mathrm{PP}_{\triangle 23}$ display the pathoplasty hypothesis, encompassing that personality (INS) affects the subsequent development of INT/EXT.

CFA was performed for each measurement occasion with the CBCL syndrome scales anxiety/depression, somatic complaints, and social withdrawal as indicators for the internalizing factor and the CBCL syndrome scales delinquent behavior and aggressive behavior as indicators for the externalizing factor. For these CFAs, we used maximum likelihood estimation with standard errors and a mean- and variance-adjusted $\chi^{2}$ test statistic that is robust to nonnormality. The latent scores for disagreeableness, emotional instability, introversion, compulsivity, internalizing, and externalizing problems were saved and used as single indicators in the multivariate LCMs for the respective factors. The error variances of these indicators were fixed to zero to identify the models. In total, four multivariate LCMs were estimated, each combining one personality factor with both internalizing and externalizing problems. In these multivariate LCMs, we controlled for age, sex, clinical status, and the covariation between internalizing and externalizing problems across time.

To examine whether a common underlying latent variable can be identified for each pair of personality symptom dimension and psychopathology dimension, item response theory
(IRT) analyses were conducted. We followed the reasoning of De Bolle et al. (2012) and included the personality symptom facets and the internalizing or externalizing syndrome scales as observed variables in these analyses, not the individual items that load on these facets or syndrome scales. Because standard IRT methods require discrete variables, the mean CBCL syndrome and DIPSI facet scores were recoded into three-category variables $^{3}$ that were in turn used for the IRT analyses.

3. Mothers were asked to rate the $\mathrm{CBCL}$ items on a scale from 0 to $2(0=n o t$ true, $1=$ somewhat or sometimes true, $2=$ very or often true). Likewise, mothers were asked to evaluate how characteristic each DiPSI item is for their child $(1=$ hardly characteristic, $2=$ little characteristic, $3=$ more or less characteristic, $4=$ characteristic, $5=$ very characteristic). These mean CBCL syndrome and DiPSI facet scores were categorized, taking into account the labels attached to the item scores and current round off rules: CBCL score $<0.50 \rightarrow$ Category $0,0.50 \leq$ CBCL score $<1.50$ $\rightarrow$ Category $1, \mathrm{CBCL}$ score $\geq 1.50 \rightarrow$ Category 2 . DiPSI score $<2.50$ $\rightarrow$ Category $0,2.50 \leq$ DiPSI score $<3.50 \rightarrow$ Category 1 , DiPSI score $\geq 3.50 \rightarrow$ Category 2 . As such, three response categories for the CBCL syndrome scores and DiPSI facet scores were obtained $(0=$ not true, $1=$ somewhat or sometimes true, $2=$ very or often true). 
All analyses were performed using Mplus, version 7 (Muthén \& Muthén, 1998-2012). In addition to $\chi^{2}$, the comparative fit index (CFI), and the standardized root mean square residual (SRMR) were reported for the CFA and the LCM analyses. For the IRT analyses, the CFI and the Tucker-Lewis index were reported. CFI and Tucker-Lewis index values above 0.90 indicate an adequate fit and values above 0.95 indicate good fit (Steiger, 1990). SRMR scores below 0.10 are considered acceptable and scores below 0.05 are indicative of good fit (Ullman, 2001). Missing data were accommodated using full information maximum likelihood under the assumption of missingness at random (cf. Allison, 2003).

\section{Results}

\section{CFAs}

The results of the CFAs on the DIPSI marker facets indicate an overall good fit for a four-factor model at $\mathrm{T} 1\left(\chi^{2}=298.23\right.$, $d f=48, p=.000 ;$ SRMR $=0.06 ;$ CFI $=0.92)$, T2 $\left(\chi^{2}=234.06, d f=48, p=.000 ;\right.$ SRMR $=0.05 ;$ CFI $=$ $0.92)$, and T3 $\left(\chi^{2}=209.44, d f=48, p=.000\right.$; SRMR $=$ 0.05 ; CFI $=0.93$ ). Completely standardized factor loadings are all significant at $p<.001$. Across the three measurement occasions, the lowest completely standardized factor loading was 0.73 for disagreeableness, 0.70 for introversion, 0.81 for emotional instability, and 0.61 for compulsivity. A two-factor model on the CBCL syndrome scales showed an overall good fit to the data for $\mathrm{T} 1\left(\chi^{2}=4.88, d f=4, p=.300\right.$; SRMR $=$ $0.01 ; \mathrm{CFI}=1.00), \mathrm{T} 2\left(\chi^{2}=6.98, d f=4, p=.137\right.$; $\mathrm{SRMR}=$ 0.02 ; CFI $=0.99)$, and T3 $\left(\chi^{2}=10.50, d f=4, p=.033\right.$; $\mathrm{SRMR}=0.02 ; \mathrm{CFI}=0.96)$. The lowest completely standardized factor loading was $0.57(p<.001)$ for internalizing problems and $0.80(p<.001)$ for externalizing problems across the three measurement occasions.

\section{Multivariate LCMs}

Results of the multivariate LCMs are summarized in Table 1. The data show overall strong support for the continuity model, because change in internalizing and externalizing problems is significantly positively related to change in each of the four maladaptive personality dimensions. Additional analyses were performed to compare the strength of the continuity associations for internalizing versus externalizing problems. More specifically, we used the $z$ statistic to compare the mean latent change correlation (i.e., averaged across change from $\mathrm{T} 1$ to $\mathrm{T} 2$ and from $\mathrm{T} 2$ to $\mathrm{T} 3$ ) between each of the maladaptive personality dimensions and internalizing versus externalizing problems, respectively. As expected, emotional instability $(z=1.8, p \leq .05)$ and introversion $(z=$ $2.61, p \leq .01)$ demonstrate stronger continuity associations with internalizing pathology than with externalizing pathology, whereas disagreeableness $(z=-2.32, p \leq .01)$ demonstrates stronger continuity associations with externalizing than with internalizing pathology. The continuity association for compul-

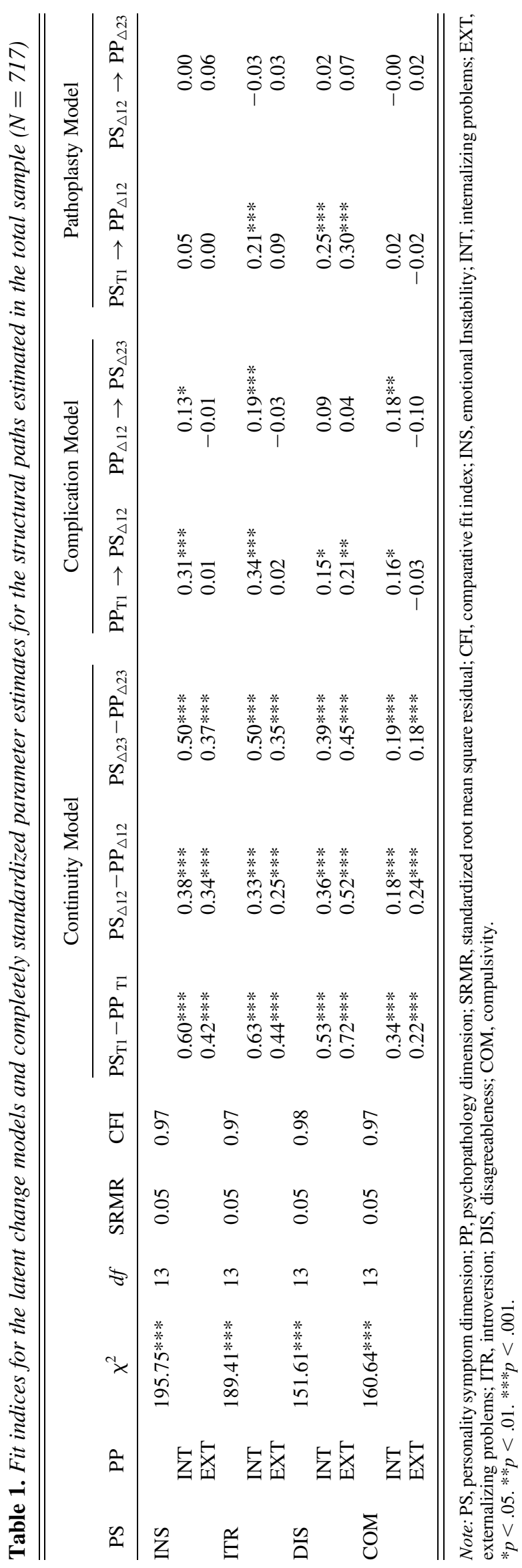


Table 2. Personality-psychopathology continuity associations from a maladaptive versus general trait perspective

\begin{tabular}{lcccc}
\hline \hline & & \multicolumn{2}{c}{ Continuity Associations } & \\
\cline { 3 - 4 } Personality & Psychopathology & Maladaptive Traits & General Traits $^{a}$ & $z$ \\
\hline \multirow{2}{*}{ N/INS } & INT & 0.44 & -0.36 & $1.80^{*}$ \\
& EXT & 0.36 & -0.23 & $2.70^{* *}$ \\
E/ITR & INT & 0.42 & -0.16 & $5.64 * * *$ \\
& EXT & 0.30 & -0.04 & $5.09 * * *$ \\
B/DIS & INT & 0.37 & -0.21 & $3.31 * *$ \\
& EXT & 0.49 & -0.40 & $2.12^{*}$ \\
C/COM & INT & 0.19 & -0.04 & $2.88^{* * *}$ \\
& EXT & 0.21 & -0.09 & $2.32^{* * *}$ \\
\hline \hline
\end{tabular}

Note: N, Neuroticism; INS, emotional instability; INT, internalizing problems; EXT, externalizing problems; E, extraversion; ITR, introversion; B, benevolence; DIS, disagreeableness; C, conscientiousness; COM, compulsivity.

${ }^{a}$ Mean continuity associations were obtained by taking the average change correlations (i.e., from Time 1 to Time 2 and from Time 2 to Time 3) from table 1 in De Bolle, Beyers, De Clercq, and De Fruyt (2012).

$* p<.05 . * * p<.01 . * * * p<.001$.

sivity and internalizing versus externalizing pathology did not significantly differ $(z=-0.39, p>.05)$.

Likewise, the strength of the continuity associations from a maladaptive versus general trait perspective was examined, by comparing the results of the present study with those of De Bolle et al. (2012). As expected, the results in Table 2 illustrate that stronger absolute continuity associations were found between maladaptive traits and psychopathology than between general traits and psychopathology.

Next to these substantial continuity associations, the results in Table 1 also show more specific complication and pathoplasty effects. Complication effects are observed from internalizing pathology to all four maladaptive personality dimensions, and from externalizing pathology to disagreeableness. More specifically, when children display or develop more severe internalizing problems, they subsequently become more introverted (i.e., they become more shy, suspicious, and display more withdrawn behavior), disagreeable (i.e., they become more impatient, aggressive, and egocen- tric), compulsive (i.e., they develop an extreme sense of achievement orientation, perfectionism, and order), and emotionally unstable (i.e., they become more dependent from others and cling more to others, ruminate more, and become more prone to feelings of anxiety), whereas the development or persistence of externally oriented behavior problems uniquely leads to subsequent higher scores on disagreeable traits.

When considering the pathoplasty effects, the results indicate that disagreeableness affects both the course of internalizing and externalizing pathology and that introversion affects the course of internalizing pathology. More specifically, children with higher scores on disagreeableness develop more (severe) internalizing and externalizing problems. In addition, as children score higher on introversion, they tend to develop more (severe) internalizing problems. The link between introversion and internalizing pathology is characterized by an exacerbating pattern: initial levels of introversion are predictive of subsequent increase in internalizing problems, which in turn results in an increasing level of introversion.

Table 3. Fit indices for confirmatory factor analysis models for T1, T2, and T3

\begin{tabular}{|c|c|c|c|c|c|c|c|c|c|c|c|c|}
\hline & \multicolumn{4}{|c|}{$\mathrm{T} 1$} & \multicolumn{4}{|c|}{$\mathrm{T} 2$} & \multicolumn{4}{|c|}{$\mathrm{T} 3$} \\
\hline & $\chi^{2}$ & $d f$ & CFI & TLI & $\chi^{2}$ & $d f$ & CFI & TLI & $\chi^{2}$ & $d f$ & CFI & TLI \\
\hline INT + INS & $124.73 * * *$ & 9 & 0.96 & 0.93 & $61.35 * * *$ & 9 & 0.98 & 0.96 & $66.26 * * *$ & 9 & 0.98 & 0.96 \\
\hline INT + ITR & $48.39 * * *$ & 9 & 0.98 & 0.97 & $46.24 * * *$ & 9 & 0.97 & 0.95 & $21.62 * *$ & 9 & 0.99 & 0.99 \\
\hline INT + DIS & $195.82 * * *$ & 9 & 0.87 & 0.78 & $69.20 * * *$ & 9 & 0.94 & 0.89 & $92.39 * * *$ & 9 & 0.93 & 0.88 \\
\hline $\mathrm{INT}+\mathrm{COM}$ & $240.31 * * *$ & 9 & 0.84 & 0.74 & $154.44 * * *$ & 9 & 0.89 & 0.82 & $115.73 * * *$ & 9 & 0.93 & 0.89 \\
\hline $\mathrm{EXT}+\mathrm{INS}$ & $56.87 * * *$ & 5 & 0.97 & 0.94 & $74.97 * * *$ & 5 & 0.96 & 0.91 & $73.76 * * *$ & 5 & 0.96 & 0.92 \\
\hline $\mathrm{EXT}+\mathrm{ITR}$ & $34.27 * * *$ & 5 & 0.97 & 0.95 & $30.74 * * *$ & 5 & 0.97 & 0.94 & $55.77 * * *$ & 5 & 0.96 & 0.92 \\
\hline $\mathrm{EXT}+\mathrm{DIS}$ & $31.48 * * *$ & 5 & 0.99 & 0.97 & $19.94 * *$ & 5 & 0.99 & 0.99 & $21.13 * * *$ & 5 & 0.99 & 0.98 \\
\hline $\mathrm{EXT}+\mathrm{COM}$ & $83.50 * * *$ & 5 & 0.93 & 0.85 & $405.82 * * *$ & 5 & 0.74 & 0.43 & $167.47 * * *$ & 5 & 0.88 & 0.75 \\
\hline
\end{tabular}

Note: T1, Time 1 first measurement; T2, Time 2 second measurement; T3, Time 3 measurement; CFI, comparative fit index; TLI, Tucker-Lewis index; INT, internalizing problems; INS, emotional instability; ITR, introversion; DIS, disagreeableness; COM, compulsivity; EXT, externalizing problems. 
Table 4. Item response theory model parameter estimates for T1, T2, and T3

\begin{tabular}{|c|c|c|c|c|c|c|c|c|}
\hline \multirow[b]{3}{*}{ Model } & \multirow{3}{*}{$\begin{array}{l}\text { Measurement } \\
\text { Occasion }\end{array}$} & \multirow[b]{3}{*}{ Construct } & \multirow{2}{*}{\multicolumn{2}{|c|}{ Discrimination }} & \multicolumn{4}{|c|}{ Difficulty } \\
\hline & & & & & \multicolumn{2}{|c|}{ Threshold 1} & \multicolumn{2}{|c|}{ Threshold 2} \\
\hline & & & $M$ & $S E$ & $M$ & $S E$ & $M$ & $S E$ \\
\hline \multirow{9}{*}{ INT + INS } & \multirow[t]{3}{*}{$\mathrm{T} 1$} & INT & 0.78 & 0.04 & 1.45 & 1.59 & 2.93 & 1.87 \\
\hline & & INS & 0.86 & 0.02 & 0.77 & 2.13 & 1.80 & 1.16 \\
\hline & & & 2.53 & & 0.36 & & 0.73 & \\
\hline & \multirow[t]{3}{*}{$\mathrm{T} 2$} & INT & 0.54 & 0.05 & 1.79 & 1.60 & 4.75 & 4.89 \\
\hline & & INS & 0.89 & 0.03 & 0.95 & 2.41 & 1.91 & 3.70 \\
\hline & & & 8.48 & & 0.41 & & 0.65 & \\
\hline & \multirow[t]{3}{*}{$\mathrm{T} 3$} & INT & 0.75 & 0.05 & 1.70 & 1.67 & 3.55 & 5.56 \\
\hline & & INS & 0.88 & 0.03 & 0.95 & 2.44 & 1.97 & 3.89 \\
\hline & & & 3.15 & & 0.36 & & 0.33 & \\
\hline \multirow[t]{9}{*}{ INT + ITR } & \multirow[t]{3}{*}{$\mathrm{T} 1$} & INT & 0.81 & 0.04 & 1.40 & 1.59 & 2.83 & 3.44 \\
\hline & & ITR & 0.87 & 0.03 & 1.40 & 2.37 & 2.35 & 4.15 \\
\hline & & & 1.70 & & 0.00 & & 0.13 & \\
\hline & \multirow[t]{3}{*}{$\mathrm{T} 2$} & INT & 0.80 & 0.05 & 1.53 & 1.56 & 3.20 & 4.79 \\
\hline & & ITR & 0.86 & 0.04 & 1.50 & 2.11 & 2.47 & 3.87 \\
\hline & & & 1.33 & & 0.02 & & 0.17 & \\
\hline & \multirow[t]{3}{*}{$\mathrm{T} 3$} & INT & 0.78 & 0.14 & 1.65 & 0.58 & 3.44 & 1.95 \\
\hline & & ITR & 0.89 & 0.03 & 1.36 & 2.69 & 2.31 & 4.79 \\
\hline & & & 1.09 & & 0.13 & & 0.31 & \\
\hline \multirow[t]{9}{*}{$\mathrm{EXT}+\mathrm{INS}$} & \multirow[t]{3}{*}{$\mathrm{T} 1$} & EXT & 0.50 & 0.08 & 2.87 & 1.08 & 4.77 & 1.99 \\
\hline & & & 0.86 & 0.03 & 0.77 & 1.82 & 1.79 & 2.68 \\
\hline & & & 5.96 & & 1.40 & & 1.26 & \\
\hline & \multirow[t]{3}{*}{$\mathrm{T} 2$} & EXT & 0.85 & 0.07 & 1.90 & 1.64 & 3.13 & 3.54 \\
\hline & & INS & 0.88 & 0.03 & 0.95 & 2.24 & 1.92 & 3.45 \\
\hline & & & 0.56 & & 0.48 & & 0.34 & \\
\hline & \multirow[t]{3}{*}{$\mathrm{T} 3$} & EXT & 0.81 & 0.06 & 1.93 & 1.76 & 3.31 & 4.54 \\
\hline & & INS & 0.87 & 0.03 & 0.98 & 2.13 & 2.00 & 3.39 \\
\hline & & & 1.26 & & 0.49 & & 0.33 & \\
\hline \multirow[t]{9}{*}{$\mathrm{EXT}+\mathrm{ITR}$} & \multirow[t]{3}{*}{$\mathrm{T} 1$} & EXT & 0.63 & 0.08 & 2.28 & 1.08 & 3.79 & 1.99 \\
\hline & & ITR & 0.86 & 0.04 & 1.42 & 1.73 & 2.38 & 3.03 \\
\hline & & & 3.64 & & 0.60 & & 0.55 & \\
\hline & $\mathrm{T} 2$ & EXT & 0.85 & 0.06 & 1.91 & 1.71 & 3.15 & 3.67 \\
\hline & & ITR & 0.85 & 0.04 & 1.51 & 2.00 & 2.50 & 3.68 \\
\hline & & & 0.00 & & 0.21 & & 0.18 & \\
\hline & $\mathrm{T} 3$ & EXT & 0.78 & 0.07 & 2.01 & 1.45 & 3.45 & 3.76 \\
\hline & & ITR & 0.89 & 0.03 & 1.37 & 2.25 & 2.33 & 4.01 \\
\hline & & & 2.04 & & 0.34 & & 0.29 & \\
\hline $\mathrm{EXT}+\mathrm{DIS}$ & $\mathrm{T} 1$ & EXT & 0.82 & 0.05 & 1.76 & 1.76 & 2.93 & 3.24 \\
\hline & & DIS & 0.79 & 0.03 & 0.70 & 1.67 & 1.88 & 2.40 \\
\hline & & & 0.73 & & 0.62 & & 0.37 & \\
\hline & $\mathrm{T} 2$ & EXT & 0.98 & 0.03 & 1.66 & 3.67 & 2.73 & 7.90 \\
\hline & & DIS & 0.79 & 0.03 & 0.91 & 1.91 & 1.94 & 2.73 \\
\hline & & & 6.33 & & 0.26 & & 0.13 & \\
\hline & T3 & EXT & 0.94 & 0.04 & 1.66 & 2.77 & 2.85 & 7.15 \\
\hline & & DIS & 0.82 & 0.03 & 0.99 & 1.94 & 2.03 & 2.91 \\
\hline & & & 3.39 & & 0.28 & & 0.15 & \\
\hline
\end{tabular}

Note: Two-tailed effect sizes for Student $t$ tests (Sloper, 2014) are in italic type. T1, Time 1 first measurement; T2, Time 2 second measurement; T3, Time 3 measurement; INT, internalizing problems; INS, emotional instability; EXT, externalizing problems; ITR, introversion; DIS, disagreeableness.

\section{IRT analyses}

The results in Table 3 show that the assumption of unidimensionality holds at all three measurement occasions for the models with internalizing problems in relation with emotional instability and introversion, and for the models with externalizing problems in relation with emotional instability, in- troversion, and disagreeableness. For these models, the discrimination and difficulty parameters are calculated and displayed in Table 4. The discrimination parameters reflect how much information the DIPSI facets and CBCL syndromes, which are included in the model, provide on average about the underlying latent factor. Most relevant to the continuity hypothesis, however, are the difficulty parameters, be- 
cause these index the location along the latent factor where the DIPSI or CBCL indicators are on average most informative from a psychometric point of view. The difficulty parameters for Threshold 1 reflect the level of the latent factor at which the likelihood of responding "a little or sometimes true" becomes higher than that of responding "not at all true." The difficulty parameters for Threshold 2 indicate the latent factor level at which the likelihood of responding "frequently to very often true" becomes higher than that of responding "a little or sometimes true." The results in Table 4 show that the difficulty parameters for Thresholds 1 and 2 are consistently higher for the CBCL indicators than for the DIPSI indicators, meaning that internalizing and externalizing problems are located at more extreme levels on the underlying latent variable than the maladaptive personality traits, consistent with the continuity hypothesis. Table 4 also displays Cohen (1988) $d$ effect sizes to compare the relative magnitude of the discrimination and difficulty parameters between the DIPSI and CBCL constructs of interest.

\section{Discussion}

The present study is the first to present a joint investigation of the continuity, pathoplasty, and complication models to explain the personality-psychopathology relations in children and adolescents from a maladaptive trait perspective, taking into account dimensional and age-specific childhood conceptualizations of maladaptive personality and psychopathology. The current work corroborates previous work of De Bolle et al. (2012) that focused on the applicability of each of these etiological models to explain personality-psychopathology relations in children and adolescents from a general trait perspective. By linking the present findings to those presented by De Bolle et al. (2012), we obtain a more comprehensive understanding of the nomological network of general personality, personality symptoms, and clinical psychopathology at young age.

In line with De Bolle et al. (2012), the current study found compelling evidence for the continuity model to explain the nature of personality-psychopathology relations, because change in each of the four personality symptom dimensions was substantially associated with change in internalizing and externalizing problem behavior. As hypothesized, the continuity associations were more robust for those personality-psychopathology associations that are conceptually closer, such as the emotional instability/introversion-internalizing problems association and the disagreeableness-externalizing problems association; and were stronger when personality was considered from a maladaptive rather than from a general trait perspective. These findings indicate that personality pathology and general mental health symptoms have common underlying factors that affect their development and course, and that link their etiology and symptom expression in complex ways (Krueger, 2005). The IRT results further support this notion, because they illustrated that these particular personality symptom-psychopathology combinations reflect the same underlying variable with internalizing and externalizing psychopathol- ogy assessing more extreme levels of this latent variable. In all, these results show the existence of two distinct internalizing and externalizing spectra that influence the strength of the specific trait-psychopathology associations, depending on whether they unfold from the same internalizing versus externalizing construct (Achenbach, 1991; Krueger \& Tackett, 2003). The findings are also consistent with those of De Clercq et al. (2009), who illustrated that childhood maladaptive traits and general internalizing and externalizing psychopathology dimensions generally show similar longitudinal patterns in terms of shape and change over time.

Although the findings demonstrate that maladaptive traits and psychopathology are mainly connected across time via nonspecific continuity associations, they also appear to have a specific reciprocal influence from a more causally oriented pathway (i.e., complication and pathoplasty effects). Overall, it can be concluded that similar etiological relations with constructs of psychopathology exist across the spectrum of general and maladaptive trait variance. More specifically, De Bolle et al. (2012) observed complication effects from externalizing behavior toward benevolence, and from internalizing behavior toward the general traits of emotional stability, conscientiousness. In the present study, we found similar complication effects from internalizing and externalizing behavior toward the more maladaptive counterparts of these traits. In addition, the present study found that internalizing behavior positively affects the developmental course of emotional instability, introversion, and compulsivity, whereas externalizing behavior appears to positively influence only the development of disagreeableness. Likewise, De Bolle et al. (2012) found pathoplasty effects from agreeableness toward both internalizing and externalizing problem behavior, whereas the present study found that disagreeableness influenced the course of both internalizing and externalizing problem behavior. The current study also found a pathoplasty effect from introversion to internalizing behavior.

Several limitations should be taken into account when interpreting the findings. First, the current study used a singleinformant perspective (i.e., both maladaptive personality and psychopathology were rated by the mothers), which can artificially inflate the continuity associations found in the current study. Future research should adopt a multiple-informant perspective to avoid inflation of parameter estimates due to shared method variance. Future studies should use alternative methods to assess the constructs of interests (e.g., observation studies) and alternative designs to pronounce upon causality (e.g., experimental designs). Second, personality and psychopathology were conceptualized at a broad level. Taking into account more fine-grained personality and psychopathology operationalizations (i.e., personality facets and psychopathology subscales, respectively) may offer additional and more specific information on the nature of the personality-psychopathology associations in children and adolescents. Third, future studies should also incorporate personality measures long before the onset of psychopathology, such that vulnerability effects can be unequivocally examined, apart from continuity, pathoplasty, and complication effects. 
From a clinical viewpoint, the current paper illustrates that maladaptive personality traits and psychopathology are closely interwoven and should therefore be considered in conjunction in clinical assessment practice. In addition, because the existence and persistence of behavioral problems may cause changes in personality at the trait level (i.e., complication effects), clinical programs should target a timely identification and treatment of behavioral problems to prevent that they evolve into anchored personality traits. In a similar vein, the existence of complication effects suggests that personality symptoms can be reduced

\section{References}

Achenbach, T. M. (1991). Manual for the Child Behavior Checklist/4-18 and 1991 profile. Burlington, VT: University of Vermont, Department of Psychiatry.

Allison, P. D. (2003). Missing data techniques for structural equation modeling. Journal of Abnormal Psychology, 112, 545-557. doi:10.1037/0021843X.112.4.545

Bienvenu, O. J., Hettema, J. M., Neale, M. C., Prescott, C. A., \& Kendler, K. S. (2007). Low extraversion and high neuroticism as indices of genetic and environmental risk for social phobia, agoraphobia, and animal phobia. American Journal of Psychiatry, 164, 1714-1721. doi:10.1176/appi.ajp.2007.06101667

Briggs-Gowan, M. J., Owens, P. L., Schwab-Stone, M. E., Leventhal, J. M., Leaf, P. J., \& Horwitz, S. M. (2003). Persistence of psychiatric disorders in pediatric settings. Journal of the American Academy of Child \& Adolescent Psychiatry, 42, 1360-1369. doi:10.1097/01.CHI.0000084834. $67701.8 \mathrm{a}$

Clark, L. A. (2005). Temperament as a unifying basis for personality and psychopathology. Journal of Abnormal Psychology, 114, 505-521.

Cohen, J. (1988). Statistical power analysis for the behavioral sciences (2nd ed.). Hillsdale, NJ: Erlbaum.

Cohen, P., Crawford, T. N., Johnson, J. G., \& Kasen, S. (2005). The Children in the Community study of developmental course of personality disorder. Journal of Personality Disorders, 19, 466-486.

Costello, E. J., Mustillo, S., Erkanli, A., Keeler, G., \& Angold, A. (2003). Prevalence and development of psychiatric disorders in childhood and adolescence. Archives of General Psychiatry, 60, 837-844.

De Bolle, M., Beyers, W., De Clercq, B., \& De Fruyt, F. (2012). General personality and psychopathology in referred and nonreferred children and adolescents: An investigation of continuity, pathoplasty, and complication models. Journal of Abnormal Psychology, 121, 958-970. doi:10.1037/a0027742

De Clercq, B., De Fruyt, F., \& Mervielde, I. (2003). Construction of the Dimensional Personality Symptom Item Pool in children (DIPSI). Ghent, Belgium: Ghent University.

De Clercq, B., De Fruyt, F., Van Leeuwen, K., \& Mervielde, I. (2006). The structure of maladaptive personality traits in childhood: A step toward an integrative developmental perspective for DSM-V. Journal of Abnormal Psychology, 115, 639-657.

De Clercq, B., De Fruyt, F., \& Widiger, T. A. (2009). Integrating a developmental perspective in dimensional models of personality disorders. Clinical Psychology Review, 29, 154-162. doi:10.1016/j.cpr.2008.12.002

De Clercq, B., Van Leeuwen, K., De Fruyt, F., Van Hiel, A., \& Mervielde, I. (2008). Maladaptive personality traits and psychopathology in childhood and adolescence: The moderating effect of parenting. Journal of Personality, 76, 357-383. doi:10.1111/j.1467-6494.2007.00489.x

De Clercq, B., Van Leeuwen, K., Van den Noortgate, W., De Bolle, M., \& De Fruyt, F. (2009). Childhood personality pathology: Dimensional stability and change. Development and Psychopathology, 21, 853-869. doi:10.1017/s0954579409000467

Dolan-Sewell, R. T., Krueger, R. F., \& Shea, M. T. (2001). Co-occurrence with syndrome disorders. In W. J. Livesley (Ed.), Handbook of personality disorders. New York: Guilford Press.

Krueger, R. F. (1999). The structure of common mental disorders. Archives of General Psychiatry, 56, 921-926.

Krueger, R. F. (2005). Continuity of Axes I and II: Toward a unified model of personality, personality disorders, and clinical disorders. Journal of Personality Disorders, 19, 233-261. through the treatment of behavioral problems. Conversely, because it has been demonstrated that personality is more amenable to change than was previously thought (Martin, Oades, \& Caputi, 2014), it might be interesting to target treatment at personality symptoms change, because this may in turn have beneficial side effects for co-occurring behavioral problems (i.e., through pathoplasty effects). Before formulating specific recommendations for the treatment of personality symptoms or behavioral problems, however, the current findings should be replicated in future research that takes into account the issues outlined above.

Krueger, R. F., Markon, K. E., Patrick, C. J., \& Iacono, W. G. (2005). Externalizing psychopathology in adulthood: A dimensional-spectrum conceptualization and its implications for DSM-V. Journal of Abnormal Psychology, 114, 537-550. doi:10.1037/0021-843x.114.4.537

Krueger, R. F., \& Tackett, J. L. (2003). Personality and psychopathology: Working toward the bigger picture. Journal of Personality Disorders, 17, 109-128.

Lenzenweger, M. F. (1999). Stability and change in personality disorder features-The longitudinal study of personality disorders. Archives of General Psychiatry, 56, 1009-1015.

Lilienfeld, S. O. (2003). Comorbidity between and within childhood externalizing and internalizing disorders: Reflections and directions. Journal of Abnormal Child Psychology, 31, 285-291.

Livesley, W. J. (2005). Introduction to the Special Issue on longitudinal studies. Journal of Personality Disorders, 19, 463-465.

Martin, L. S., Oades, L. G., \& Caputi, P. (2014). Intentional personality change coaching: A randomized control trial of participant selected personality facet change using the five-factor model of personality. International Coaching Psychology Review, 9, 182-195.

McCrae, R. R., \& Costa, P. T. J. (2008). The five-factor theory of personality. In O. P. John, R. W. Robins, \& L. A. Pervin (Eds.), Handbook of personality: Theory and research (3rd ed., pp. 159-181). New York: Guilford Press.

Mineka, S., Watson, D., \& Clark, L. A. (1998). Comorbidity of anxiety and unipolar mood disorders. Annual Review of Psychology, 49, 377-412.

Muthén, L. K., \& Muthén, B. O. (1998-2012). Mplus user's guide (7th ed.). Los Angeles: Author.

Roza, S. J., Hofstra, M. B., van der Ende, J., \& Verhulst, F. C. (2003). Stable prediction of mood and anxiety disorders based on behavioral and emotional problems in childhood: A 14-year follow-up during childhood, adolescence, and young adulthood. American Journal of Psychiatry, 160, 2116-2121.

Skodol, A. E., Gunderson, J. G., Shea, M. T., McGlashan, T. H., Morey, L. C., Sanislow, C. A., et al. (2005). The Collaborative Longitudinal Personality Disorders Study (CLPS): Overview and implications. Journal of Personality Disorders, 19, 487-504.

Steiger, J. H. (1990). Structural model evaluation and modification: An interval estimation approach. Multivariate Behavioral Research, 25, 173-180.

Tackett, J. L. (2006). Evaluating models of the personality-psychopathology relationship in children and adolescents. Clinical Psychology Review, 26, 584-599.

Ullman, J. B. (2001). A first course in structural equation modeling. Applied Psychological Measurement, 25, 101-102. doi:10.1177/ 01466216010251008

Verhulst, F. C., Van der Ende, J., \& Koot, H. M. (1996). Handleiding voor de CBCL/4-18 [Manual of the CBCL/4-18]. Rotterdam: Universiteit Rotterdam, Afdeling Kinderen Jeugdpsychiatrie.

Widiger, T. A. (2003). Personality disorder and Axis I psychopathology: The problematic boundary of Axis I and Axis II. Journal of Personality Disorders, 17, 90-108.

Widiger, T. A., \& Simonsen, E. (2005). Alternative dimensional models of personality disorder: Finding a common ground. Journal of Personality Disorders, 19, 110-130.

Zanarini, M. C., Frankenburg, F. R., Hennen, J., Reich, D. B., \& Silk, K. R. (2005). The McLean Study of Adult Development (MSAD): Overview and implications of the first six years of prospective follow-up. Journal of Personality Disorders, 19, 505-523. 